Reprod. Nutr. Dévelop. 1980, 20 (1 A), 61-76.

\title{
Effets de l'hypophysectomie puis de l'administration de la gonadotropine hCG sur le taux de testostérone plasmatique et sur la structure de l'épididyme et des glandes accessoires chez le verrat (Sus scrofa L.)
}

par Michelle MORAT, A. LOCATELLI *, M. TERQUI *, Michelle CHEVALIER, Monique CHAMBON, J. P. DUFAURE

Equipe de Cytophysiologie, Laboratoire de Biologie Cellulaire ef Génétique, Université de Clermont-Ferrand II, B. P. 45, 63170 Aubière

* Station de Physiologie de la Reproduction, I.N.R.A., Nouzilly 37380 Monnaie.

Summary. Plasma festosterone level and structure of the epididymis and accessory organs of the boar (Sus scrofa L.) after hypophysectomy followed by hCG gonadotropin administration.

Hypophysectomy of the boar resulted in a rapid drop in plasma testosterone level. hCG injections ( $400 \mathrm{IU} /$ day) considerably elevated testosteronemia (up to $16.41 \mathrm{ng} / \mathrm{ml}$ ). This effect was obtained either in the days following the operation or several months after (up to 134 days). In the latter case, the effect was temporary because the testosterone level dropped again after 15 days of injections. The sexual target organs (epididymis, seminal vesicle, prostate and Cowper glands) underwent structural change which, in the epithelium, corresponded to fluctuations in testosterone level. These effects were less marked in the cauda epididymis than in other target tissues. In various target organs, the conjunctivomuscular stroma developed after the operation ; it did not regress at all, or incompletely, when the epithelia were stimulated indirectly by hCG, and began to hypertrophy when the hCG was no longer effective. Variations in stromal reaction were organ-dependent. When the hCG was administered for more than 14 days, it seemed to have a desensitizing effect ; these mechanisms have been discussed.

\section{Introduction.}

Les conséquences de l'hypophysectomie sur la structure ef le fonctionnement du testicule sont connues depuis longtemps (Smith, 1930 ; voir également revues dans Albert, 1961 ef Morat, 1977a). Elles se manifestent, en particulier, par une chute du taux des androgènes consécutive à une action directe de la gonadotropine LH (ICSH) sur les cellules de Leydig (Squire et al., 1963). De ce fait, les organes accessoires du tractus génital mâle qui sont contrôlés par la testostérone subissent une atrophie comme dans le cas d'une castration (revue dans Price ef Williams-Ashman, 1961). 
Mais il a en outre été signalé que, pour la prostate, la régression peut être plus accentuée chez les hypophysectomisés que chez les castrés (Huggins et Russel, 1946) et différents auteurs ont avancé l'idée que des hormones antéhypophysaires comme la prolactine et l'hormone de croissance pouvaient agir en synergie avec la testostérone pour maintenir les glandes accessoires (revue dans Price et Williams-Ashman 1961).

Dans le cadre du travail entrepris sur le contrôle des cellules de Leydig du testicule de verrat, nous avons montré que l'hypophysectomie conduit à une atrophie mais non à une disparition des cellules de Leydig (Dufaure ef al., 1974 ; Morat 1977a). L'administration de la gonadotropine $\mathrm{hCG}$ in vivo ou in vitro permet de stimuler ces cellules (Dufaure et al., 1974 ; Morat, 1977b). Dans les conditions in vitro, les cellules de Leydig redeviennent fonctionnelles puisqu'elles sécrèłent de la testostérone qui a été mise en évidence par RIA (radioimmuno assay) (Morat ef al., 1978). Mais cette stimulation n'est que transitoire car après 15 jours de traitement les cellules de Leydig s'atrophient à nouveau (Dufaure et al., 1974 ; Morat, 1977b) et la production de testostérone cesse (Moral ef al., 1978). Nous étudions ici les variations de la testostéronémie consécutives à l'hypophysectomie et au traitement par hCG des animaux opérés. Les variations du taux de testostérone sont mises en parallèle avec des changements de structure histologique de différents organes accessoires : corps de l'épididyme, vésicules séminales, prostate, glandes de Cowper.

\section{Matériel et méthodes.}

Les travaux ont été effectués sur des verrats pubères (Sus scrofa L.) de race Large White âgés de 7 à 8 mois et hypophysectomisés suivant la technique de du Mesnil du Buisson ef al., (1964).

Les prélèvements sanguins et les injections d'hCG ont été réalisés chez trois animaux. Le sang périphérique est prélevé à différents intervalles compris entre 2 et 14 jours au moyen d'un cathéłer de polyéthylène stérile inséré dans une veine marginale auriculaire et maintenu à demeure. Les animaux hypophysectomisés depuis 134 jours (verrat $n^{\circ} 1$ ) et 107 jours (verrat $n^{\circ} 2$ ) ont reçu une injection quotidienne de 500 Ul d'hCG (Organon) respectivement pendant 17 jours et 46 jours. Le verrat $n^{\circ} 3$ a reçu 500 Ul d'hCG le $6^{\mathrm{e}}$ jour et le $9 \mathrm{e}$ jour après l'hypophysectomie ; il a été abattu 86 jours après l'hypophysectomie.

Le sang recueilli sur héparine a été centrifugé à froid ef le plasma conservé à - $15^{\circ} \mathrm{C}$. La testostéronémie a été déterminée par un dosage radioimmunologique direct dans le plasma selon le principe proposé par Galloway et al. (1974). Un tel dosage direct a été validé chez le verrat (Meusy-Dessole, 1974). Le dosage a été réalisé dans les conditions (antisérum, immunoprécipitation) définies par Garnier et al. (1978). La sensibilité de ce dosage est comprise entre 0,1 et $0,2 \mathrm{ng}$ par $\mathrm{ml}$ pour une prise d'essai de $0,05 \mathrm{ml}$.

Les organes sexuels accessoires ont été prélevés à l'abattage de différents animaux normaux, hypophysectomisés, hypophysectomisés traités par hCG pendant 17 jours et 46 jours. Ils sont fixés dans le Bouin Hollande sublimé, inclus en paraffine en passant par le butanol et débités en coupes de $7 \mu \mathrm{m}$ d'épaisseur. Les coupes ont été colorées par le glychémalun-éosine, l'Azan de Heidenhain ou le Mallory. L'état du tissu interstitiel leydigien est connu pour tous ces cas et a fait l'objet de publications 
séparées (Morat, 1977a et b). Les mesures servant à caractériser l'état histologique des organes accessoires ont été effectuées d'après des clichés photographiques $\times 100$ ef $\times 400$. Dans chaque cas, 20 unités prises au hasard sont mesurées sur différents clichés et le chiffre donné correspond à la moyenne suivie de la valeur de l'écart-type.

\section{Résultats.}

1. Testostérone plasmatique. - Les variations de la testostéronémie consécutives à l'ablation de l'hypophyse ont été suivies chez le verrat $n^{\circ} 3$ (fig. 1). Deux jours après l'opération, le taux d'hormone est de $1,85 \mathrm{ng} / \mathrm{ml}$, après 4 jours il est de $0,92 \mathrm{ng} / \mathrm{ml}$. En prenant comme référence chez le pubère normal un taux hormonal moyen de $4 \mathrm{ng} / \mathrm{ml}$ (Meusy-Dessole 1975), on constate que la disparition de l'hormone est rapide. A la fin de l'expérience, après 86 jours, le taux est très faible $(29 \mathrm{pg} / \mathrm{ml})$.

L'injection d'hCG dans les jours qui suivent l'hypophysectomie provoque une brusque élévation de la testostéronémie qui atteint des valeurs élevées $(11,09 \mathrm{ng} / \mathrm{m} \mathrm{l}$ après la 1 re injection; $11,02 \mathrm{ng} / \mathrm{ml}$ après la $2 \mathrm{e}$ ) (fig. 1). Des administrations d'hCG

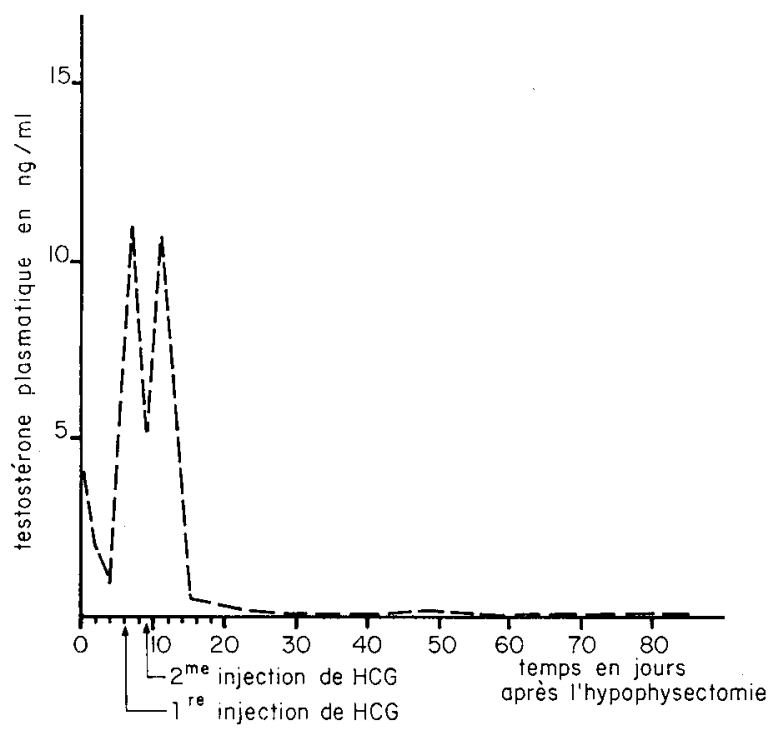

FIG. 1.

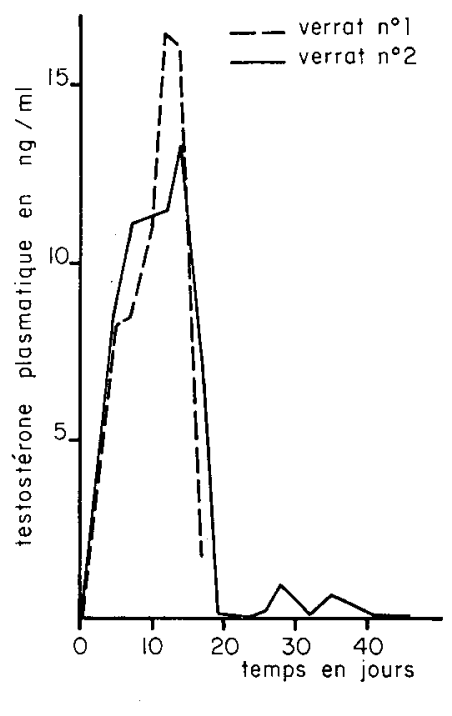

FIG. 2 .

FIG. 1. - Variations du taux de festostérone plasmatique chez un verrot hypophysectomisé ef effets de l'injection d'hCG dans les jours qui suivent l'hypophysectomie. L'animal a reçu deux injections de $500 \mathrm{Ul} d^{\prime h} \mathrm{CG}$, le $6^{\mathrm{e}}$ et le $9^{\mathrm{e}}$ jour après l'opération. Noter une chute rapide de la testostéronémie consécutive à l'opération suivie d'une augmentation très importante sous l'action de la gonadotropine dont l'effet disparaît très vite. La valeur au jour $0(4 \mathrm{ng} / \mathrm{ml})$ est donnée d'après MeusyDessole (1975).

FIG. 2. - Variations du taux de festostérone plasmatique chez deux verrats hypophysectomisés depuis 134 jours $\left(n^{\circ} 1\right)$ et 107 jours ( $n^{\circ} 2$ ), soumis à une injection quotidienne de 500 Ul d'hCG respectivement pendant 17 et 46 jours. Le taux de testostérone augmente progressivement jusqu'au 14e jour puis chute après le $14^{\mathrm{e}}$ jour ; ce taux demeure inférieur à $1 \mathrm{ng} / \mathrm{ml}$ jusqu'à la fin de l'expérience. 
effectuées sur des animaux hypophysectomisés depuis 107 jours et 134 jours (fig. 2) entraînent une élévation progressive de la testostéronémie qui atteint les valeurs de $16,41 \mathrm{ng} / \mathrm{ml}$ après 12 jours d'injections chez le verrat no 1 et $13,25 \mathrm{ng} / \mathrm{ml}$ après 14 jours chez le verrat $n^{\circ} 2$. Au-delà de 14 jours, les taux d'hormone circulante baissent brusquement chez les deux animaux bien que les injections soient prolongées. La testostéronémie, suivie jusqu'au 46e jour d'injections chez le verrat no 2 se maintient ensuite à une valeur inférieure à $1 \mathrm{ng} / \mathrm{ml}$ avec des variations importantes suivant les prélèvements, comprises entre 40 ef $980 \mathrm{pg} / \mathrm{ml}$ ).

2. Effecteurs sexuels. - Chez le mâle adulte ou pubère normal, la structure de ces différents organes est typique et correspond à des descriptions déjà données dans la littérature, pour la plupart chez les Rongeurs (voir Price and Williams-Ashman, 1961 ; Bloom and Fawcett, 1968 ; Narbaitz, 1974). L'étude histologique porte sur plusieurs animaux normaux, un verrat hypophysectomisé depuis 86 jours ( $n^{\circ} 3$ ), un verrat hypophysectomisé depuis 134 jours puis traité pendant 17 jours par hCG (no 1 ), un verrat hypophysectomisé depuis 107 jours puis traité pendant 46 jours par hCG (verrat $\left.n^{\circ} 2\right)$.

a) Corps de l'épididyme (PI. I, fig. 1 à 8). L'aspect histologique du tissu normal est illustré dans les figures 1 et 2 et différentes données morphométriques rapportées dans le tableau 1. L'épithélium n'offre aucune particularité remarquable. Le tissu intertubulaire (stroma) est formé, 1) par environ 4 assises de cellules musculaires lisses

\section{PLANCHE 1}

Structure histologique du corps de l'épididyme.

FIG. 1 et 2. - Verrat normal de 8 mois. Fig. 1 : les tubes épithéliaux sont tassés les uns contre les autres avec un tissu conjonctif intertubulaire peu abondant et une mince gaine musculaire péritubulaire (flèche) ; $\times 90$. Fig. 2 : détail de la structure de l'épithélium ; noter dans les cellules principales l'abondance des stéréocils et la forme presque sphérique des noyaux ; $\times 366$.

FIG. 3 et 4 . - Verrat hypophysectomisé depuis 86 jours. Fig. 3 : les tubes, de diamètre réduit, sont séparés par un abondant tissu conjonctif ; les assises musculaires (flèche) se sont épaissies; $\times 90$. Fig. 4 : la hauteur de l'épithélium a diminué ef le cytoplasme est plus lacunaire ; les noyaux cellulaires ont pris une forme ovale; les stéréocils sont toujours présents mais moins nombreux ; $\times 366$.

FIG. 5 et $6 .-$ Verrat hypophysectomisé depuis 134 jours puis traité pendant 17 jours par hCG. Fig. 5 : le diamètre des tubes a augmenté ; le tissu intertubulaire, dont la gaine musculaire (flèche), reste épais ; $\times 90$. Fig. 6 : l'épithélium est élevé et a développé de nombreuses microvillosités (stéréocils) ; dans les cellules principales, les noyaux restent de forme allongée du fait d'une faible croissance la cellule en largeur, mais on trouve à la base de l'épithélium une assise de cellules de remplacement à noyaux sphériques : $\times 366$.

FIG. 7 ef 8. - Verrat hypophysectamisé depuis 107 jours puis traité pendant 46 jours par hCG. Fig. $7:$ le diamètre des tubes est réduit ; $\times 90$. Fig. 8 : l'épithélium a fortement régressé ; l'absence apparente de stéréocils est un artefact de la préparation ; le conjonctif juxia-tubulaire est abondant (double flèche) : $\times 366$. 

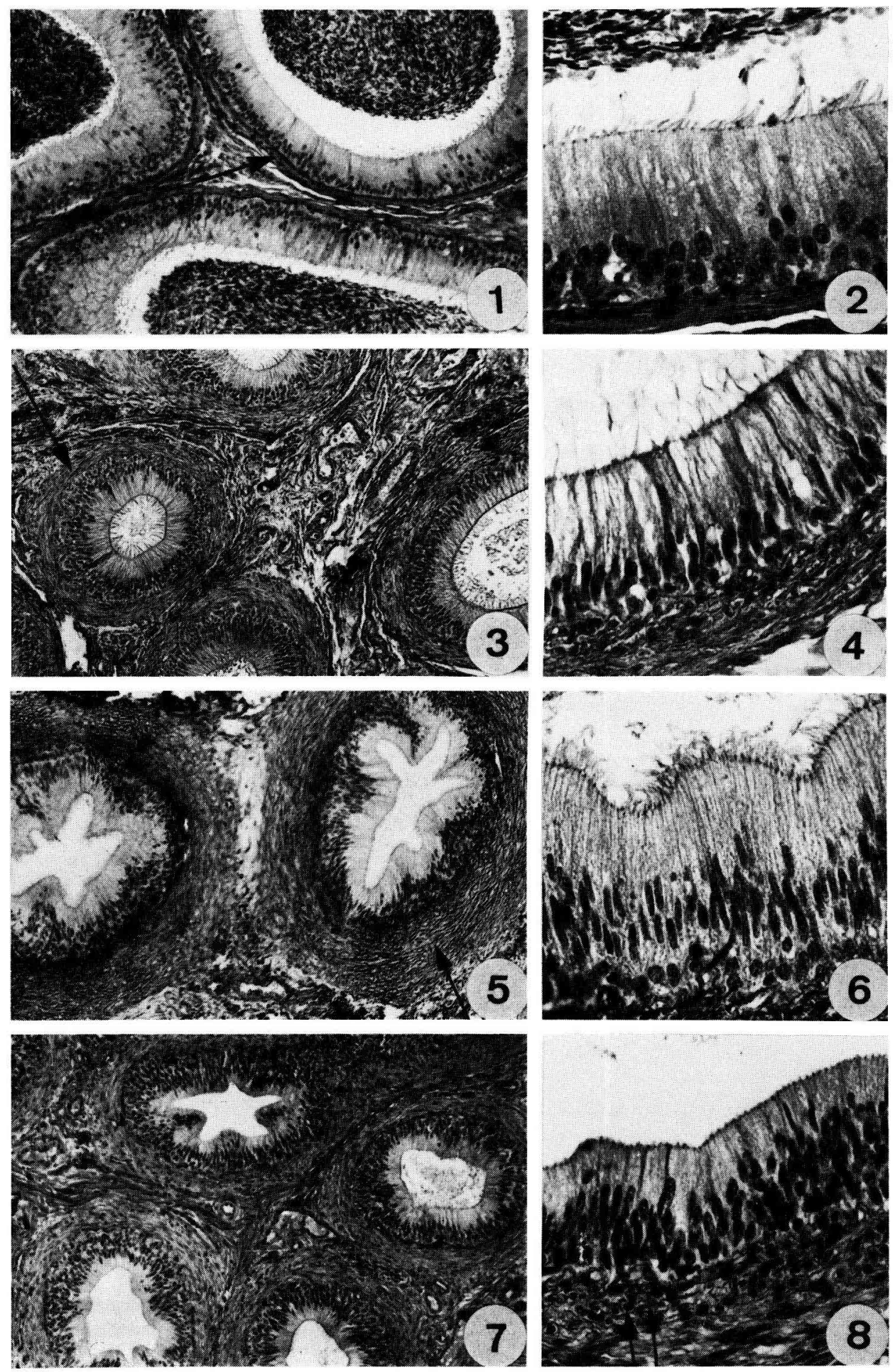
TABLEAU 1

Données morphométriques sur les structures accessoires de l'appareil génital du verrat

\begin{tabular}{|c|c|c|c|c|c|c|}
\hline & & & $\begin{array}{l}\text { Hauteur de } \\
\text { l'épithélium }\end{array}$ & $\begin{array}{l}\text { Taux de } \\
\text { variation }\end{array}$ & $\begin{array}{l}\text { Taille des } \\
\text { noyaux } \\
\text { épithéliaux }\end{array}$ & \\
\hline & & $\begin{array}{l}\text { Diamètres } \\
\text { des tubes }\end{array}$ & & & & $\begin{array}{l}\text { Epaisseur } \\
\text { des gaines } \\
\text { musculaires }\end{array}$ \\
\hline \multirow[t]{5}{*}{ Epididyme } & Normal & $555,0 \pm 15,9$ & $84,50 \pm 4,62$ & 1,41 & $\left\{\begin{array}{l}9,25 \pm 0,30 \\
5,87 \pm 0,29\end{array}\right.$ & $13,37 \pm 0,80$ \\
\hline & Hypophysectomisé & $239,0 \pm 6,5$ & $59,75 \pm 3,05$ & \multirow[t]{4}{*}{1,46} & $\left\{\begin{aligned} 11,25 & \pm 0,41 \\
2,95 & \pm 0,13\end{aligned}\right.$ & $39,50 \pm 1,59$ \\
\hline & \multirow[t]{2}{*}{ Hyp. + 17 j hCG } & $358,5 \pm 13,7$ & $87,50 \pm 6,80$ & & \multirow{2}{*}{$\begin{array}{r}12,62 \pm 0,43 \\
2,75 \pm 0,18 \\
6,00 \pm 0,27\end{array}$} & $61,25 \pm 3,23$ \\
\hline & & & & & & \\
\hline & Hyp. +46 j hCG & $181,5 \pm 8,1$ & $47,50 \pm 4,20$ & & $\left\{\begin{array}{r}11,70 \pm 0,33 \\
4,50 \pm 0,22\end{array}\right.$ & $55,50 \pm 3,50$ \\
\hline $\begin{array}{l}\text { Vésicules } \\
\text { séminales }\end{array}$ & $\begin{array}{l}\text { Normal } \\
\text { Hypophysectomisé } \\
\text { Hyp. + } 17 \text { j hCG } \\
\text { Hyp. + } 46 \text { j hCG }\end{array}$ & & $\left.\begin{array}{l}42,77 \pm 2,33 \\
13,25 \pm 1,01 \\
32,50 \pm 1,85 \\
12,25 \pm 0,87\end{array}\right\}$ & $\begin{array}{l}3,23 \\
2,46\end{array}$ & & \\
\hline \multirow[t]{3}{*}{ Prostate } & $\begin{array}{l}\text { Normal } \\
\text { Hypophysectomisé }\end{array}$ & & $\begin{aligned} 20,38 & \pm 0,68 \\
8,62 & \pm 0,52\end{aligned}$ & \multirow{2}{*}{$\begin{array}{l}2,37 \\
1,74\end{array}$} & \multirow{2}{*}{$\begin{array}{l}7,00 \pm 0,22 \\
6,25 \pm 0,35 \\
3,12 \pm 0,23 \\
6,12 \pm 0,21\end{array}$} & \\
\hline & Hyp. $+17 j$ hCG & & $15,00 \pm 0,65$ & & & \\
\hline & & $\begin{array}{l}\text { Diamètre } \\
\text { des acini }\end{array}$ & & & & \\
\hline \multirow[t]{4}{*}{$\begin{array}{l}\text { Glandes de } \\
\text { Cowper }\end{array}$} & Normal & $82,00 \pm 3,98$ & $30,50 \pm 1,74$ & 2,62 & $\left\{\begin{array}{l}5,77 \pm 0,35 \\
2,52 \pm 0,23\end{array}\right.$ & \\
\hline & Hypophysectomisé & $26,00 \pm 2,19$ & $11,67 \pm 0,92$ & \multirow[t]{2}{*}{1,82} & $\left\{\begin{array}{l}5,75 \pm 0,42 \\
3,25 \pm 0,30\end{array}\right.$ & \\
\hline & Hyp. +17 jhCG & $60,00 \pm 3,30$ & $21,25 \pm 1,50$ & & $\left\{\begin{array}{l}6,80 \pm 0,25 \\
3,25 \pm 0,30\end{array}\right.$ & \\
\hline & Hyp. +46 j hCG & $24,00 \pm 1,32$ & $10,40 \pm 0,91$ & & $\left\{\begin{array}{l}5,92 \pm 0,30 \\
3,40 \pm 0,28\end{array}\right.$ & \\
\hline
\end{tabular}

Les chiffres donnés en $\mu \mathrm{m}$ correspondent à des moyennes affectées de l'écart-type. Pour les noyaux généralement de forme ovoïde, on a réuni sous une accolade la mesure du plus grand et du plus petit diamètre. Remarquer qu'il y a deux sortes de noyaux dans l'épididyme d'hypophysectomisé traité 17 jours par hCG (cellules principales et cellules basales). Il n'y a pas de différences significatives portant sur la taille des noyaux épithéliaux dans les différentes conditions expérimentales. On note néanmoins, un allongement des noyaux après l'hypophysectomie dans les cellules principales de l'épididyme et dans la prostate. Les taux de variation dans la hauteur des épithéliums ont été calculés en faisant le rapport entre les hauteurs chez le normal et chez l'hypophysectomisé (taux de diminution) et le rapport entre les hauteurs chez le traité pendant 17 jours par hCG et l'hypophysectomisé (taux d'augmentation). 
entourant les tubes et séparées de ces derniers par une très mince couche conjonctive, 2) par un tissu conjonctif intertubulaire peu développé ef à prédominance de fibres collagènes. Chez l'hypophysectomisé, le diamètre des tubes diminue de plus de la moitié (tabl. 1). La hauteur des épithéliums est également réduite et on note un allongement des noyaux ainsi qu'une diminution de la densité des stéréocils (microvillosités) à l'apex des cellules. La gaine musculaire s'est épaissie (6 à 7 assises) et des éléments conjonctifs se sont infiltrés entre l'épithélium et les assises musculaires (conjonctif juxta-tubulaire). Le tissu conjonctif intertubulaire à prédominance collagène a augmenté de volume. Après un traitement par hCG pendant 17 jours, le diamètre des fubes s'accroît mais sans atteindre une valeur normale (tabl. 1). La hauteur de l'épithélium devient au contraire très importante par suite, en particulier, d'une prolifération des cellules basales de remplacement (cellules à noyau sphérique, fig. 6). Les stéréocils redeviennent abondants. Cette stimulation du tissu épithélial contraste avec une absence d'effet dans les tissus conjonctivo-musculaires qui continuent à s'épaissir, en particulier au niveau de la gaine musculaire (fig. 5). Cette dernière, très développée (fabl. 1), paraît contrarier l'expansion du tissu épithélial. La poursuite du traitement par hCG se traduit par une atrophie de l'épithélium dont les stéréocils se raréfient alors que les tissus conjonctivo-musculaires restent fortement développés (fig. 7), le conjonctif juxta-tubulaire continuant à s'épaissir (fig. 8 , double flèche).

b) Vésicules séminales (PI. II, fig. 9 à 16). Elles ont une constitution typique chez l'animal normal : grandes alvéoles (fig. 9) bordées par un épithélium unistratifié formant des replis. Les cellules épithéliales manifestent une activité sécrétoire intense de type apocrine (fig. 10). De minces travées (stroma) séparent les alvéoles : on y observe surtout des fibres collagènes et quelques cellules musculaires lisses. L'hypophysectomie entraîne une réduction de la hauteur de l'épithélium (tabl. 1 ef fig. 12) et un développement considérable du stroma (fig. 11 et 12). Dans ce dernier, on distingue : un conjonctif sous épithélial dense et riche en collagène (fig. 12), un tissu plus lâche comprenant un mélange de fibres collagènes ef de cellules musculaires lisses ef enfin un axe conjonctif à prédominance collagène. Après 17 jours de stimulation par hCG, la hauteur des cellules épithéliales est accrue (tabl. 1 ef fig. 14) ef des signes d'activité sécrétoire sont manifestes. Le développement et la disposition des tissus conjonctivo-musculaires ne changent pas. La prolongation du traitement par hCG se traduit par un nouvel effondrement épithélial (tabl. 1 et fig. 16) alors que le stroma reste développé : on note même un épaississement du tissu musculaire lisse (fig. 15 , flèche.

c) Prostate (PI. III, fig. 17 à 22). Cet organe est mal individualisé chez le verrat (Price and Williams-Ashman, 1961) mais sa structure est banale. Le tissu épithélial, peu élevé (tabl. 1 et fig. 18), forme des alvéoles et des acini noyés dans un stroma lâche formé d'éléments conjonctifs avec fibres collagènes et de cellules musculaires lisses ; ces tissus sont disposés en lobules séparés par des cloisons conjonctivo-musculaires (fig. 17). Chez l'hypophysectomisé, la hauteur de l'épithélium diminue fortement (tabl. 1). L'extrême réduction de la taille des cellules est bien visible sur la fig. 20. Le stroma conjonctivo-musculaire occupe un volume important. II y a peu de changements (faible épaississement) au niveau des cloisons conjonctivo-musculaires qui 

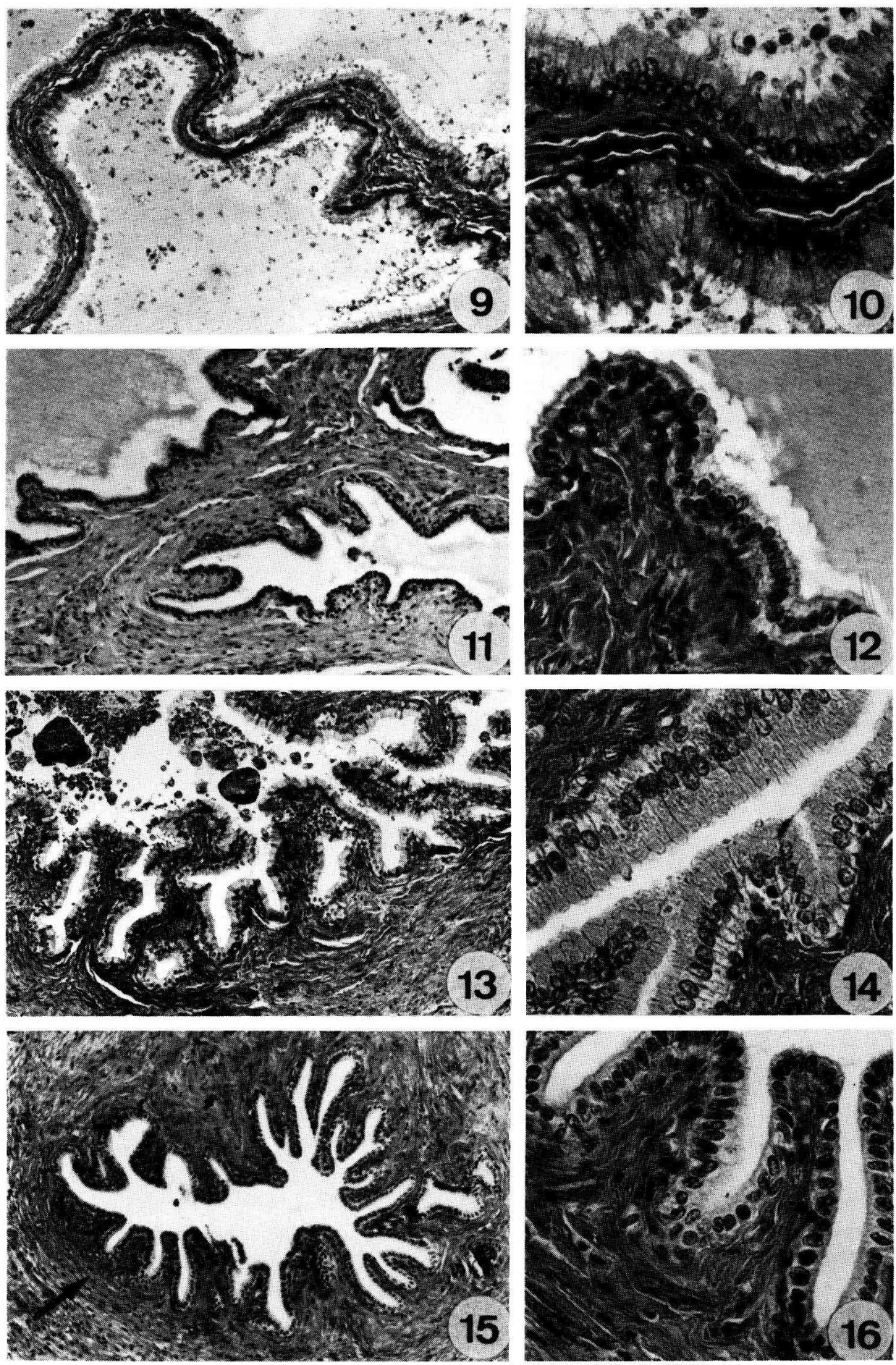
séparent les lobules. Après 17 jours de traitement par hCG, la reprise de l'épithélium est nette (tabl. 1 et fig. 22) et le volume du stroma diminue de manière sensible (fig. 21) mais l'organe ne retrouve pas tout à fait une structure normale. La prostate n'a pas été étudiée après une action prolongée d'hCG.

d) Glandes de Cowper (PI. IV, fig. 23 à 30). Ces glandes particulièrement volumineuses chez le verrat sont découpées en lobules séparés par des travées de tissu conjonctif très riche en collagène. De grands faisceaux de fibres musculaires lisses sont inclus dans la capsule conjonctive qui entoure l'organe. Chez l'animal normal (fig. 23 et 24), l'épithélium glandulaire forme des acini tassés les uns contre les autres, ne laissant que peu d'espace pour le tissu conjonctif sous-jacent extrêmement réduit ef dont les éléments n'ont pas pu être analysés. La glande a une structure muqueuse typique (fig. 24). Après hypophysectomie, l'épithélium s'atrophie (tabl. 1 ef fig. 26) tandis que se développe un tissu conjonctif lâche entre les acini (fig. 25 et 26) mais ce sont surtout les travées conjonctives interlobulaires qui s'hypertrophient (non figuré ici). Un traitement pendant 17 jours par hCG entraîne une reprise d'activité sécrétoire dans l'épithélium (fig. 28) qui ne retrouve cependant pas les caractères morphométriques de l'animal normal (tabl. 1). Corrélativement, le tissu conjonctif qui entoure les acini diminue de volume alors que les travées interlobulaires restent assez développées. Après 46 jours de traitement, l'épithélium s'atrophie à nouveau (tabl. 1 et fig. 30) sans que l'on assiste à un nouveau développement du conjonctif sous jacent : il y a un tassement des acini qui doif traduire une contraction générale de la glande.

\section{PLANCHE II}

Structure histologique des vésicules séminales.

FIG. 9 et 10. - Verrat normal de 8 mois. Fig. 9 : l'organe est constitué par la juxtaposition de grandes alvéoles séparées par de fines travées de stroma ; $\times 90$. Fig. 10 : détail de la paroi des alvéoles; les cellules épithéliales, de forme cylindrique, déversent dans la lumière des éléments figurés ; $\times 366$.

FIG. 11 et 12. - Verrat hypophysectomisé depuis 86 jours. Fig. 11 : noter la diminution de la taille des alvéoles ef un important développement du stroma ; $\times 90$. Fig. 12 : la hauteur des cellules épithéliales est réduite, les éléments figurés ont disparu ; $\times 366$.

FIG. 13 et 14. - Verrat hypophysectomisé depuis 134 jours puis traité pendant 17 jours par hCG. Fig. 13 : la taille des alvéoles a augmenté et les replis de la paroi se sont approfondis pour donner des cryptes; la lumière confient beaucoup d'éléments figurés; noter la persistance d'un stroma abondant ; $\times 90$. Fig. 14 : détail de l'épithélium ; $\times 366$.

FIG. 15 et 16. - Verrat hypophysectomisé depuis 107 jours puis traité pendant 46 jours par hCG. Fig. 15 : la taille des alvéoles est réduite alors que le stroma occupe un volume important avec un développement particulier des assises musculaires péri-alvéolaires (flèche) $; \times 90$. Fig. 16 : le cytoplasme des cellules épithéliales est réduif : $\times 366$. 

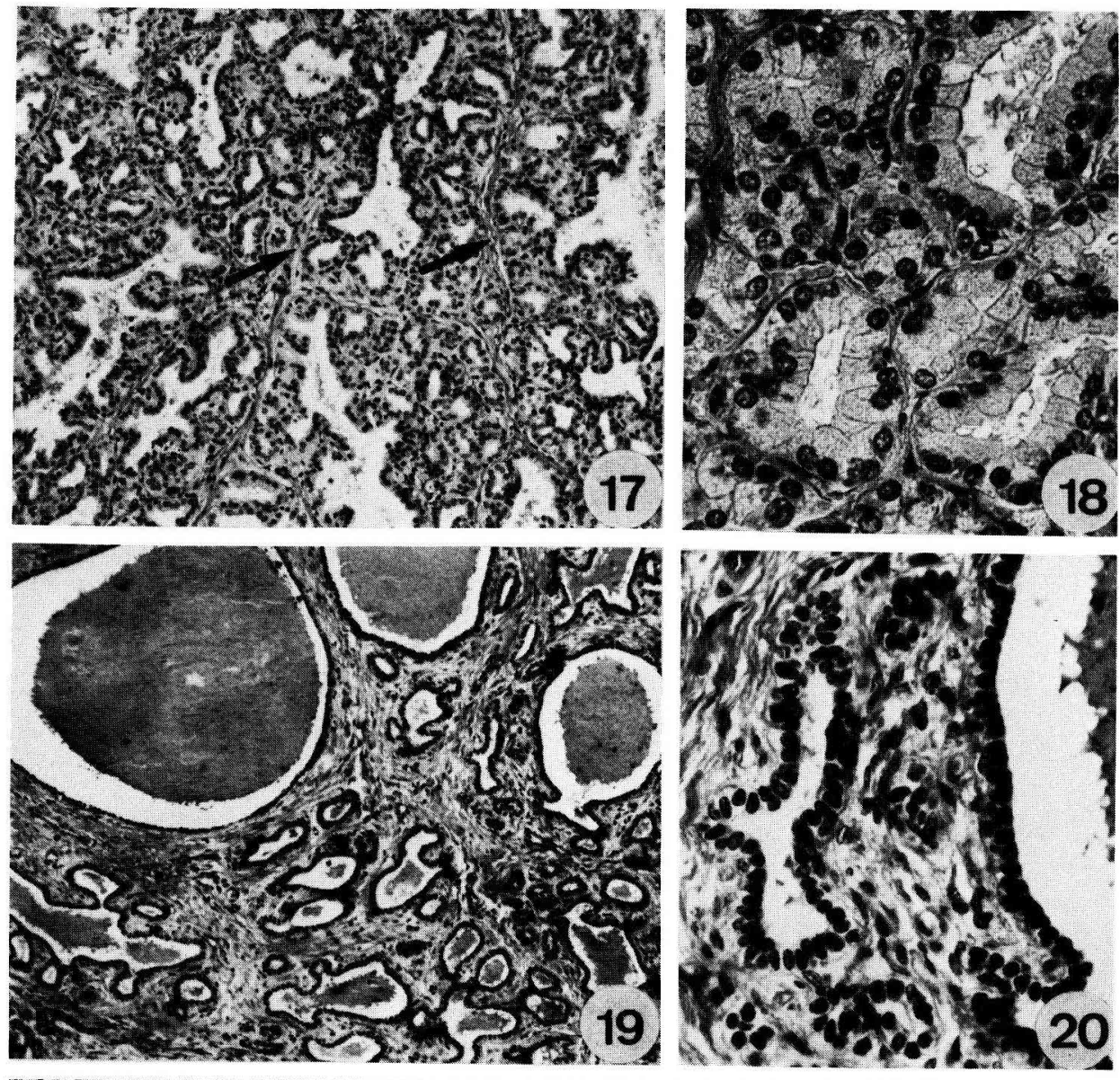

by

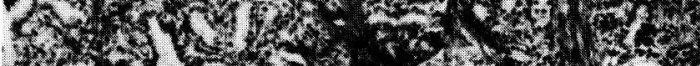

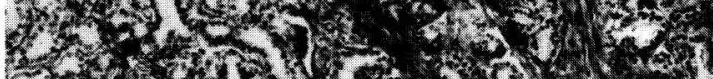

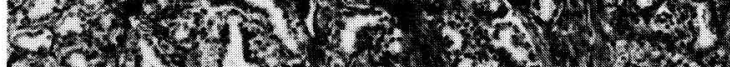

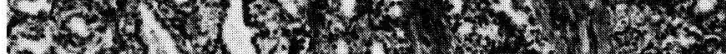

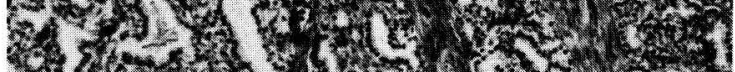

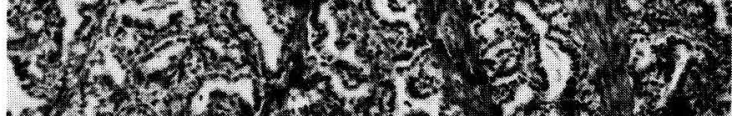

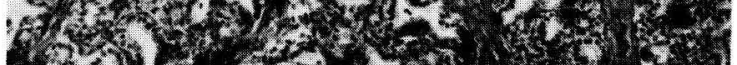

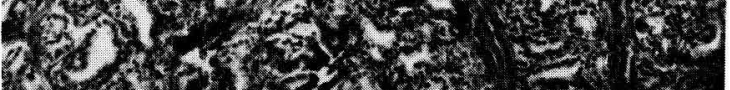
6. -

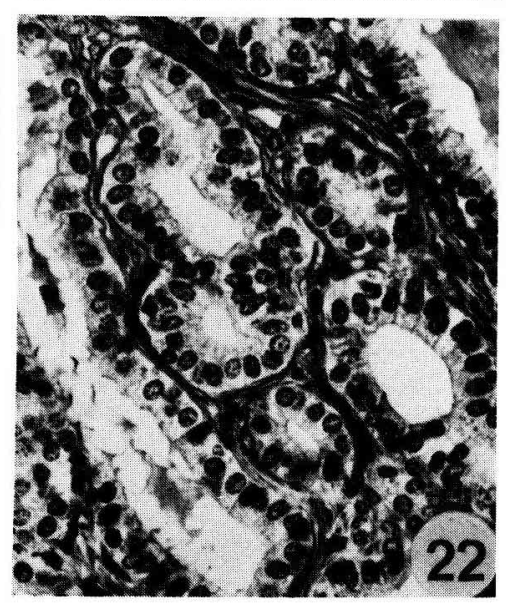




\section{Discussion.}

Il est maintenant bien connu que la gonadotropine hypophysaire LH agit sur le testicule pour réguler la synthèse de festostérone dans les cellules de Leydig (voir Hall, 1970). On peut, en particulier, obtenir chez l'hypophysectomisé une sécrétion d'hormones androgènes par l'administration d'une gonadotropine purifiée (Lostroh ef al., 1958). L'hypophysectomie du verrat se traduit par une chute très rapide du taux de testostérone plasmatique qui est à mettre en corrélation avec une atrophie poussée des cellules de Leydig (Dufaure et al., 1974 ; Morat, 1977a). L'administration de la gonadotropine hCG stimule fortement la synthèse de testostérone non seulement dans les jours qui suivent l'hypophysectomie, mais également chez l'animal opéré depuis plusieurs mois. Ce résultat est conforme à nos précédentes observations puisque nous avons montré que les cellules de Leydig ne disparaissent pas chez le verrat hypophysectomisé, qu'elles réacquièrent une ultrastructure de cellules à stéroïdes fonctionnelles lorsqu'elles sont stimulées par hCG (Dufaure et al., 1974 ; Morat, $1977 b$ ) ef que dans des conditions de culture organotypique elles produisent de la testostérone en présence d'hCG (Morat ef al., 1978) ; elles répondent également à des LH de différentes origines (Morat, en préparation).

L'effet d'hCG cesse brusquement après 14 jours d'injections. Ce phénomène a également été observé en étudiant la morphologie des cellules de Leydig (Dufaure et al., 1974 ; Morat, 1977b). Il survient de la même façon in vitro (Morat, 1977b). Il peut s'agir d'un phénomène analogue à la désensibilisation des cellules de Leydig du rat découvert par Hsueh ef al. (1976) et par Sharpe (1976). Remarquons toutefois que les

\section{PLANCHE III}

Structure histologique de la prostate.

FIG. 17 et 18. - Verrat normal de 8 mois. Fig. 17 : la glande cloisonnée par de longues assises conjonctivo-musculaires (flèche) est formée d'alvéoles dans lesquelles débouchent des acini ; les alvéoles aboutissent à des conduits évacuateurs qui ne sont pas compris dans le champ; $\times 90$. Fig. 18 : structure de l'épithélium glandulaire des acini ; les cellules ne sont pas très élevées et leur noyau sphérique est situé en position basale ; $\times 366$.

FIG. 19 et 20. - Verrat hypophysectomisé depuis 86 jours. Fig. 19 : région mixte comprenant les formations alvéolo-acineuses ef les conduits évacuateurs; les structures épithéliales sont réduites alors que le stroma occupe un volume important; la lumière des alvéoles et des conduits renferme des restes de sécrétion ; $\times 90$. Fig. 20 : détail montrant la réduction extrême du cytoplasme des cellules épithéliales ; les noyaux sont devenus ovoïdes ; $\times 366$.

FIG. 21 et 22. - Verrof hypophysectomisé depuis 134 jours puis traité pendant 17 jours par hCG. Fig. 21 : les structures épithéliales sont redevenues prépondérantes, le stroma ayant régressé ; $\times 90$. Fig. 22 : détail de l'épithélium glandulaire montrant que les noyaux sont redevenus sphériques et que le volume du cyłoplasme a augmenté ; le stroma est réduit mais plus abondant qu'avant I'hypophysectomie : $\times 366$. 


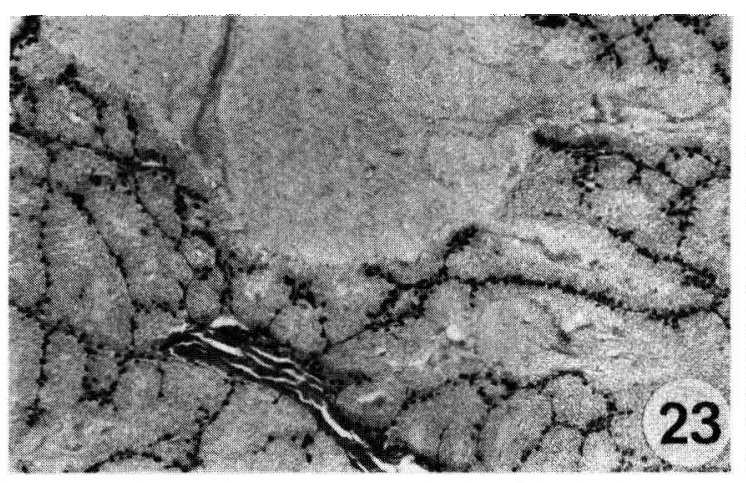

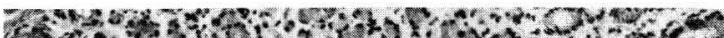

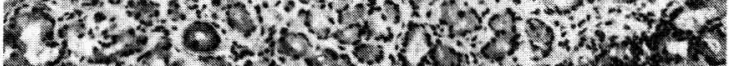

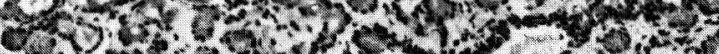

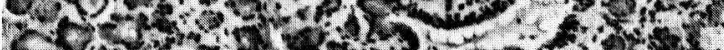

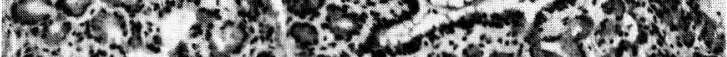

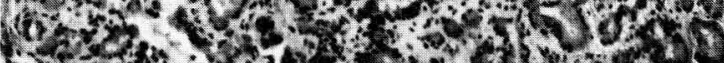

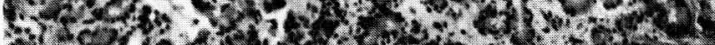

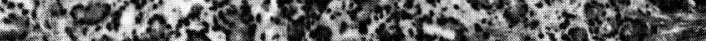

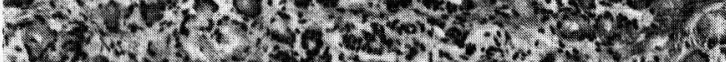
2.

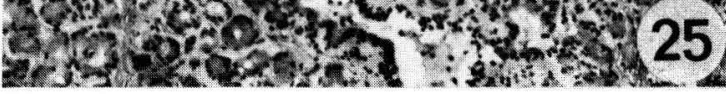

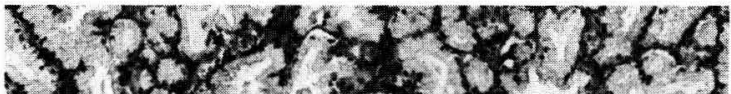
b.

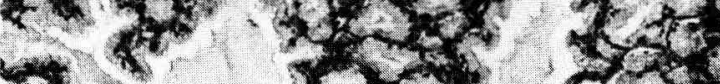

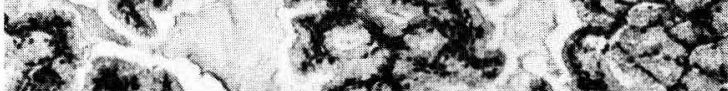

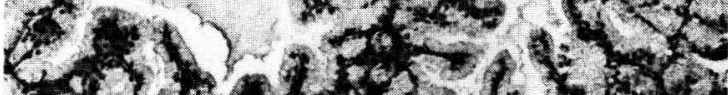

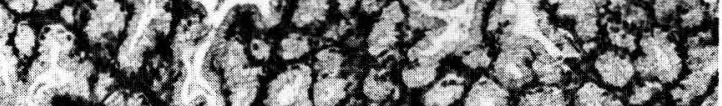

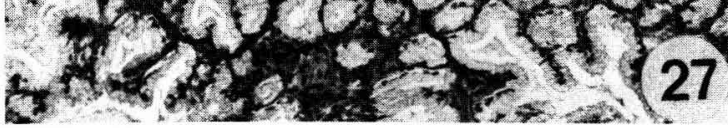

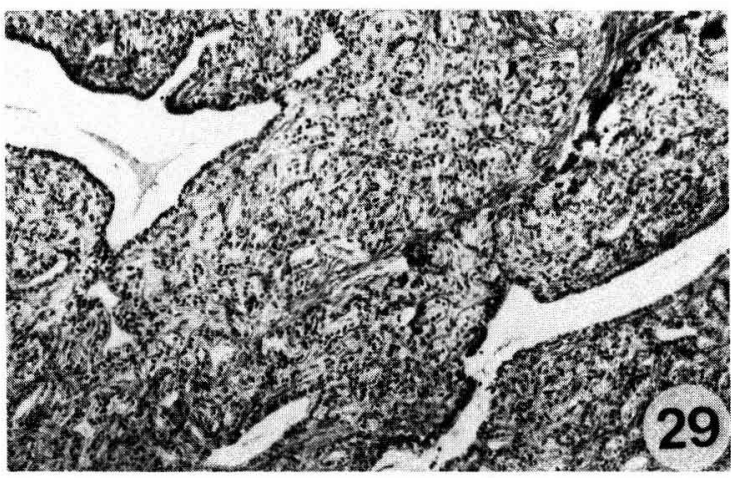

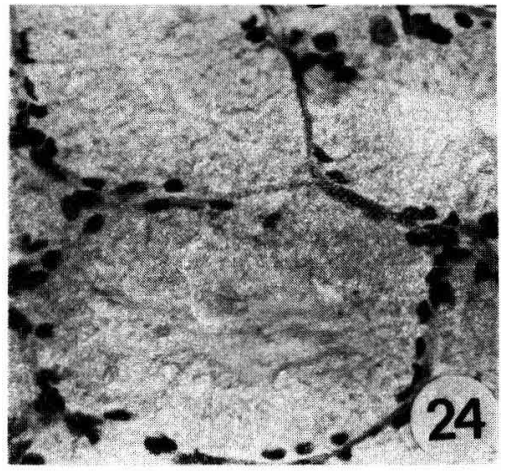
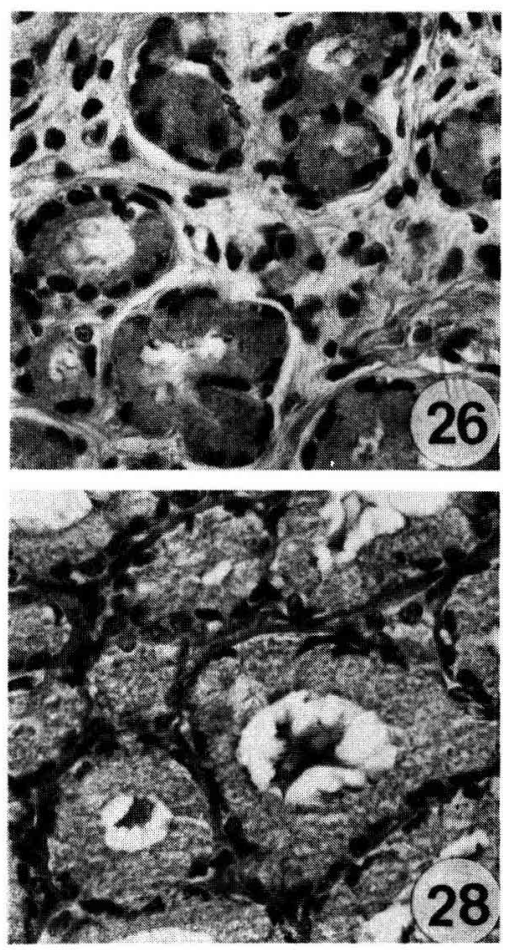

The

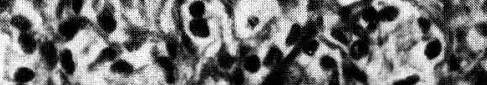
- in,

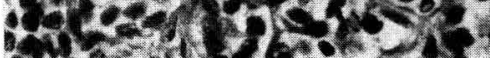

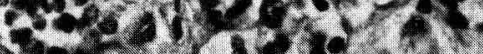

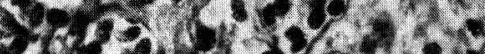

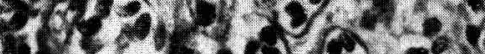

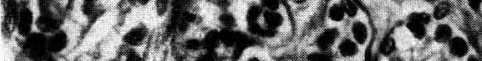

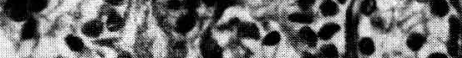

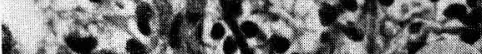
1. 130 
conditions d'apparition du phénomène ne sont pas les mêmes, la désensibilisation des cellules de Leydig du rat étant un phénomène aigu ef réversible survenant dans les heures qui suivent l'administration de gonadotropine. Comme pour la désensibilisation, on peut, outre la perte des récepteurs membranaires (« down-regulation ») envisager différents autres mécanismes : découplage récepteur-adényl cyclase (Saez ef al., 1978) déviation dans la chaîne de biosynthèse des stéroïdes au-delà de la formation de l'AMPc (Cigorraga et al., 1978). Un effet inhibiteur direct de la production de testostérone induit par l'œstradiol a également été envisagé (Cigorraga et al., 1978 ; Haour ef Saez, 1978).

Dans les différentes conditions expérimentales, la réponse des effecteurs sexuels est conforme au taux de testostérone circulante. Ce serait toutefois une simplification que de conclure qu'il s'agit d'un effet de castration. Il est connu que des hormones hypophysaires non gonadotropes peuvent agir directement sur les effecteurs sexuels, en particulier la prolactine et l'hormone de croissance (Lostroh et $\mathrm{Li}, 1957$ et revue dans Price and Williams-Ashman, 1961). La question du synergisme de la prolactine a été réétudiée (Grayhack et Leibowitz, 1967 ; Resnick et al., 1974) mais reste un des points obscurs du modèle concernant le mécanisme d'action des androgènes (Mainwaring, 1977). En absence d'une étude parallèle des effets de la castration, nos expériences ne permettent pas d'apporter d'éléments sur ce point.

L'épithélium des glandes accessoires est un bon indicateur des taux d'androgènes (Cavazos, 1975). Il en est ainsi chez le verrat. Les variations de la hauteur des épithéliums tiennent à des variations du volume cytoplasmique. Les volumes nucléaires ne subissent pas de changements significatifs (tabl. 1). Les seules modifications nucléai-

\section{PLANCHE IV}

Structure histologique des glandes de Cowper.

FIG. 23 et 24. - Verrot normal de 8 mois. Fig. 23 : la glande est formée de lobules séparés par des travées de tissu conjonctif ; chaque lobule contient de nombreux acini serrés les uns contre les autres et quelques sections de grands conduits évacuateurs; $\times 90$. Fig. 24 : détail des acini montrant l'aspect typique d'une glande muqueuse ; le tissus intertubulaire est réduit à l'extrême ; $\times 366$.

FIG. 25 et 26. - Verrat hypophysectomisé depuis 86 jcurs. Fig. 25 : les acini de diamètre très réduit sont noyés dans un abondant tissu conjonctif ; $\times 90$. Fig. 26 : détail de la glande; la structure reste de type muqueux, seul le volume cellulaire est réduit ; $\times 366$.

FIG. 27 et 28. - Verrat hypophysectomisé depuis 134 jours puis traité pendant 17 jours par hCG. Fig. 27 : vue générale montrant la stimulation de la glande (augmentation de taille des acini et réduction de volume du stroma) qui ne retrouve pas tout à fait un aspect normal ; $\times 90$. Fig. 28 : détail de l'épithélium glandulaire; $\times 366$.

FIG. 29 et 30. - Verrat hypophysectomisé depuis 107 jours puis traité pendant 46 jours par hCG. Fig. 29 : l'effondrement de la structure glandulaire est perceptible à un faible grossissement; le tissu conjonctif n'a pas recommencé à s'hypertrophier (comparer avec la fig. 25) ; $\times 90$. Fig. 30 : l'involution de l'épithélium glandulaire est importante car on ne reconnaît plus une glande de type muqueux ; $\times 366$. 
res notées à cette échelle sont des changements de forme (allongement) dans le cas de l'épididyme et de la prostate, qui peuvent s'expliquer par des causes mécaniques (compression due à une contraction de la cellule). La sensibilité des épithéliums est variable suivant les organes, ce qui avait déjà été noté (voir Cavazos, 1975). Chez le verrat, d'après les calculs des faux de diminution et des taux d'augmentation des épithéliums (tabl. 1), ce sont les vésicules séminales qui répondent le mieux, puis les glandes de Cowper, la prostate et enfin l'épididyme. En général, le taux d'augmentation est plus faible que le taux de diminution (stimulation incomplète) ; dans l'épididyme la situation est particulière puisque l'augmentation globale de la hauteur de l'épithélium est due en partie au développement d'une assise de cellules de remplacement (PI. I, fig. 6). L'épithélium de l'épididyme répond moins que celui des glandes accessoires du tractus génital ; les résultats obtenus sur ce tissu sont conformes aux observations très complètes d'Orgebin-Crist et Davies (1974) effectuées chez le lapin.

Les variations au niveau du stroma conjonctivo-musculaire des organes accessoires des Mammifères n'ont pas été très étudiées, ce qui est regrettable. La privation en androgènes se traduit, en général, par une augmentation du volume du stroma et cette évolution esf réversible lorsque des androgènes sont à nouveau disponibles (voir pour la prostate Baulieu ef al., 1975). Il nous est apparu chez le verrat que les phénomènes sont assez complexes car les réponses ne sont pas les mêmes suivant les organes et suivant les tissus (tissu conjonctif et tissu musculaire lisse par exemple). Le tissu musculaire lisse normalement présent autour des formations épithéliales dans l'épididyme et les vésicules séminales s'hyperplasie après l'hypophysectomie et ne rétrocède pas ou peu après stimulation par hCG. Le tissu conjonctif juxta-tubulaire qui se développe dans l'épididyme à la suite de l'hypophysectomie continue à s'hypertrophier sous l'effet ef après cessation de l'effet d'hCG. Dans la prostate et les glandes de Cowper, le tissu conjonctif lâche sous-épithélial suit au contraire mais en sens inverse les variations de l'épithélium (augmentation de volume à la privation hormonale et diminution lors de la supplémentation). Au contraire, les travées de collagène qui délimitent les lobules s'hypertrophient de manière irréversible, au moins dans les glandes de Cowper. Il nous est difficile de conclure car notre échantillonnage est très réduit, mais les faits rapportés sont nets et mériteraient une étude approfondie. Il est possible que les androgènes produits par le testicule d'hypophysectomisé ou par le testicule traité par hCG (voir, plus haut, discussion sur la désensibilisation) ne soient pas tout à fait les mêmes que chez l'animal normal. Il a été montré également (Lacroix et al., 1978) qu'une administration prolongée d'hCG tant chez le normal que chez l'hypophysectomisé entraîne une déviation dans le métabolisme intratesticulaire de la testostérone. En fonction de ce métabolisme, il est possible que l'on obtienne un effet différent sur l'épithélium et sur le stroma. Par exemple, Baulieu ef al. (1975) ont indiqué que l'androstanolone ( $5 \alpha$-DHT) stimule l'épithélium mais ne fait pas régresser le stroma dans la prostate de rat cultivée in vitro. L'étude, en cours, de l'évolution des profils hormonaux $\left(\Delta_{4}\right.$ androstenedione, testostérone et $5 \alpha$-DHT) lors de la réactivation des cellules de Leydig in vifro, apportera peut être un élément de réponse à cette question. 


\section{References}

ALBERT A., 1961. The mammalian testis, 305-365. In YOUNG W. C., Sex and internal secretions, The Williams and Wilkins Co., Baltimore.

BAULIEU E. E., LE GOASCOGNE C., GROYER A., FEYEL-CABANES T., ROBEL P., 1975. Morphological and biochemical parameters of androgen effects on rat ventral prostate in organ culture. Vitamins Hormones, 33, 1-38.

BLOOM W., FAWCETT DON W., 1968. A fext book of histology. 9th edition. W. B. Saunders Co., Philadelphia, London, Toronto.

CAVAZOS L. F., 1975. Fine structure and function correlates of male accessory sex glands of rodents, 353-381. In ASTWOOD V. E. B., GREEP R. O., Handbook of Physiology, 5, sect. 7. Endocrinology. Am. Physiol. Soc., Washington, D. C.

CIGORRAGA S. B., DUFAU M. L., CATT K. J., 1978. Regulation of luteinizing hormone receptors and steroidogenesis in gonadotropin-desensitized Leydig cells. J. biol. Chem., 253, 4297-4304.

DUfAURE J. P., DU MESNIL DU BUISSON F., MORAT M., CHEVALIER M., LOCATELLI A., 1974. Effets de l'hypophysectomie et de l'administration d'hormone gonadotrope (hCG) sur les cellules de Leydig du testicule de verrat. C. R. Acad. Sci. Paris, Sér. D, 279, 1907-1910.

DU MESNIL DU BUISSON F., LÉGLISE P. C., CHODKIEWICZ J. P., 1964. Technique de I'hypophysectomie par voie transfrontale sus-orbitaire chez le porc. Ann. Biol. anim. Biochim. Biophys., 4, $229-237$.

GALLOWAY D. B., COTTA Y., PELLETIER J., TERQUI M., 1974. Circulating luteinizing hormone and testosterone in rams after luteinizing releasing hormone treatment. Acta endocrinol., $\mathbf{7 7}$, $1-9$.

GARNIER D. H., COTTA Y., TERQUI M., 1978. Androgen radioimmunoassay in the ram : results of direct plasma testosterone and dehydroepiandrosterone measurement and physiological evaluation. Ann. Biol. anim. Bioch. Biophys., 18, 265-287.

GRAYHACK J. T., LEIBOVITZ J. M., 1967. Effect of prolactin on the citric acid and lateral lobe of the prostate of Sprague-Dawley rats. Invest. Urol., 5, 87-94.

HALL. P. F., 1970. Endocrinology of the testis, 1-71. In JOHNSON A. D., GOMES W. R., VANDEMARK N. L., The testis, Acad. Press, New York and London.

HAOUR F., SAEZ J. M., 1978. Leydig-cell responsiveness to LH-hCG stimulation : mechanisms of hCG- and steroid-induced refractoriness, 497-516. In : Mc KERNS K. W., Structure and function of the gonadotropins. Plenum Publ. Co.

HSUEH A. J. W., DUFAU M. L., CATT K. J., 1976. Regulation of luteinizing hormone receptors in testicular interstitial cells by gonadotropin. Biochem. Biophys. Res. Commun., 72, 1145-1152.

HUGGINS C., RUSSELL P. S., 1946. Quantitative effects of hypophysectomy on testis and prostate of dogs. Endocrinology, 39, 1-7.

LACROIX E., EECHAUTE W., VAN NIMMEN D., 1978. Influence of prolonged hCG administration on testicular steroids of hypophysectomized rats. Ann. Endocrinol. (Paris), 39, 161-162.

LOSTROH A. J., LI C. H., 1957. Stimulation of the sex accessories of hypophysectomized male rats by non-gonadotrophic hormones of the pituifary gland. Acta endocrinol., 25, 1-16.

LOSTROH A. J., SQUIRE P. G., LI C. H., 1958. Bioassay of interstitial cell-stimulating hormone in the hypophysectomized male rat by the ventral prostate test. Endocrinology, 62, 833-842.

MAINWARING W. I. P., 1977. The mechanism of action of androgens. Monogr. Endocrinol., 10, Springer Verlag, New York, Heidelberg, Berlin.

MEUSY-DESSOLE N., 1974. Evolution de la testostérone plasmatique au cours de la vie fœtale chez le pore domestique (Sus Scrofa L.). C. R. Acad. Sci., Paris, sér. D, 278, 1260-1263.

MEUSY-DESSOLE N., 1975. Variations quantitatives de la testostérone plasmatique chez le Porc mâle, de la naissance à l'âge adulte. C. R. Acad. Sci. Paris, sér. D, 281, 1875-1878.

MORAT M., 1977a. Action morphogène des hormones gonadotropes sur les cellules de Leydig du testicule de verrat. I. Effets de l'hypophysectomie. Arch. Anat. micr. Morph. exp., 66, 119-142.

MORAT M., 1977b. II. Effets de l'administration de gonadotropine chorionique après hypophysectomie. Action in vivo et en culture organotypique. Arch. Anat. micr. Morph. exp., 66, 181-205. 
MORAT M., CHEVALIER M., DUFAURE J. P., 1978. Mise en évidence d'une sécrétion de testostérone par le tissu testiculaire de verrat hypophysectomisé, sous l'action de hCG, en culture organotypique. C. R. Acad. Sci. Paris, sér. D, 286, 1605-1608.

NARBAITZ R., 1974. Embryology, anatomy and histology of the male sex accessory glands, 3-15. In BRANDES D., Male accessory sex organs, Acad. Press Inc., New York, San Francisco, London.

ORGEBIN-CRIST M. C., DAVIES J., 1974. Functional and morphological effects of hypophysectomy and androgen replacement in the rabbit epididymis. Cell Tiss. Res., 148, 183-201.

PRICE D., WILLIAMS-ASHMAN H. G., 1961. The accessory reproductive glands of mammals, 366448. In YOUNG W. C., Sex and internal secretions, the Williams and Wilkins Co., Baltimore.

RESNICK M. I., WALVOURD D. J., GRAYHACK J. T., 1974. Effect of prolactin on testosterone uptake by the perfused canine prostate. Surg. Forum, 25, 70-72.

SAEZ J. M., HAOUR F., CATHIARD A. M., 1978. Early hCG-induced desensitization in Leydig cells. Biochem. Biophys. Res. Commun., 72, 1145-1152.

SHARPE R. M., 1976. hCG-induced decrease in availability of rat testis receptors, Nature (London). 264, 644-646.

SMITH P. E., 1930. Hypophysectomy and a replacement therapy in the rat. Am. J. Anat., 45, 205-273.

SQUIRE P. G., JOHNSTON R. E., LYONS W. R., 1963. Intratesticular injections of interstitial cellstimulating hormones in hypophysectomized rats. Int. J. Fertil., 8, 531-536. 